\title{
STABILISATION OF HIGH ASPECT RATIO MIXED FINITE ELEMENTS FOR INCOMPRESSIBLE FLOW
}

\author{
MARK AINSWORTH, GABRIEL R. BARRENECHEA, AND ANDREAS WACHTEL
}

\begin{abstract}
Anisotropically refined mixed finite elements are beneficial for the resolution of local features such as boundary layers. Unfortunately, the stability of the resulting scheme is highly sensitive to the aspect ratio of the elements. Previous analysis revealed that the degeneration arises from a relatively small number of spurious (piecewise constant) pressure modes. The present article is concerned with resolving the problem of how to suppress the spurious pressure modes in order to restore stability yet at the same time not incurring any deterioration in the approximation properties of the reduced pressure space.

Two results are presented. The first gives the minimal constraints on the pressure space needed to restore stability with respect to aspect ratio and shows that the approximation properties of the constrained pressure space and the unconstrained pressure space are essentially identical. Alternatively, one can impose the constraint weakly through the use of a stabilised finite element scheme. A second result shows that the stabilised finite element scheme is robust with respect to the aspect ratio of the elements and produces an approximation that satisfies an error bound of the same type to the mixed finite element scheme using the constrained space.
\end{abstract}

\section{Introduction and Main Result}

Consider Stokes equations on a polygonal domain $\Omega \subset \mathbb{R}^{2}$

$$
-\Delta \boldsymbol{u}+\operatorname{grad} p=\boldsymbol{f}, \quad \operatorname{div} \boldsymbol{u}=0 \text { in } \Omega
$$

subject to $\boldsymbol{u}=\mathbf{0}$ on $\partial \Omega$ and $\langle p\rangle_{\Omega}=0$, where $\langle p\rangle_{\omega}$ denotes the mean value of $p$ over $\omega \subset \Omega$, and $\boldsymbol{f} \in L^{2}(\Omega)$ is a given source term. Throughout, we use standard notations for Sobolev spaces [1].

The variational form of (1) consists of seeking $\boldsymbol{u} \in \boldsymbol{V}=H_{0}^{1}(\Omega)$ and $p \in M=$ $L_{0}^{2}(\Omega)$ such that

$$
B(\boldsymbol{u}, p ; \boldsymbol{v}, q)=(\boldsymbol{f}, \boldsymbol{v})_{\Omega} \text { for all }(\boldsymbol{v}, q) \in \boldsymbol{V} \times M
$$

where

$$
B(\boldsymbol{u}, p ; \boldsymbol{v}, q)=(\operatorname{grad} \boldsymbol{u}, \operatorname{grad} \boldsymbol{v})_{\Omega}-(\operatorname{div} \boldsymbol{v}, p)_{\Omega}-(\operatorname{div} \boldsymbol{u}, q)_{\Omega} .
$$

The well-posedness of problem (2) is a consequence of the inf-sup condition [9, pp. $58-61]$ :

$$
\inf _{q \in M} \sup _{\boldsymbol{v} \in \boldsymbol{V}} \frac{(\operatorname{div} \boldsymbol{v}, q)_{\Omega}}{|\boldsymbol{v}|_{H^{1}(\Omega)}\|q\|_{\Omega}}=\beta_{\Omega}>0
$$

This result is established, for example, in [8, Lemma III.3.1].

1991 Mathematics Subject Classification. 65N12, 65N30, 65N50.

Key words and phrases. anisotropic meshes, stabilisation, spurious pressure mode.

Partial support for MA under AFOSR contract FA9550-12-1-0399 is gratefully acknowledged.

The work of GRB and AW was supported by the Leverhulme Trust under grant RPG-2012-483. 


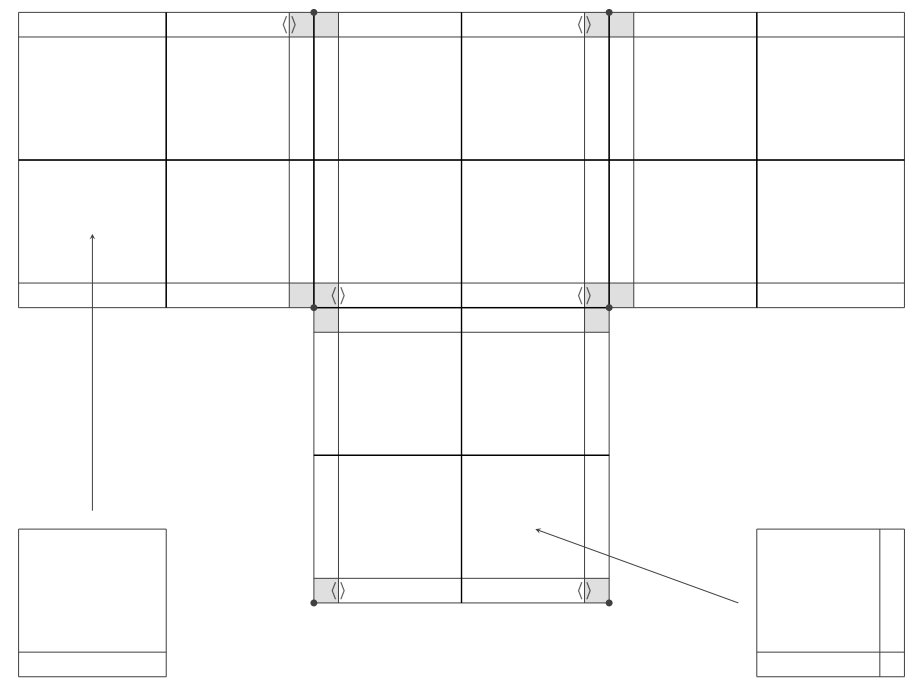

(a) Edge patch

(b) Corner patch

Figure 1. A typical example of an anisotropically refined mesh of the type considered in the present work.

Let $\mathcal{P}$ be a conforming partition of $\Omega$ into the union of closed parallelograms such that the non-empty intersection of distinct elements $K$ and $K^{\prime}$ is either a single common point or a single common edge of both elements. For $k \geq 1$, a mixed finite element approximation of the Stokes problem is sought using the spaces

$$
\boldsymbol{V}_{\mathcal{P}}=\left\{\boldsymbol{v} \in \boldsymbol{V}: \boldsymbol{v} \circ F_{K} \in \mathbb{Q}_{k+1}^{2} \quad \forall K \in \mathcal{P}\right\}
$$

and

$$
M_{\mathcal{P}}=\left\{q \in M: q \circ F_{K} \in \mathbb{P}_{k-1} \quad \forall K \in \mathcal{P}\right\}
$$

where $F_{K}:[0,1]^{2} \rightarrow K \in \mathcal{P}$ is an invertible affine transformation.

The pair $\boldsymbol{V}_{\mathcal{P}} \times M_{\mathcal{P}}$ is known $[5,14]$ to satisfy a discrete version of (4) with a positive constant $\beta_{\mathcal{P}}$. As a consequence, there exists a unique mixed finite element approximation $\boldsymbol{u}_{\mathcal{P}} \in \boldsymbol{V}_{\mathcal{P}}$ and $p_{\mathcal{P}} \in M_{\mathcal{P}}$ such that

$$
B\left(\boldsymbol{u}_{\mathcal{P}}, p_{\mathcal{P}} ; \boldsymbol{v}, q\right)=(\boldsymbol{f}, \boldsymbol{v})_{\Omega} \text { for all }(\boldsymbol{v}, q) \in \boldsymbol{V}_{\mathcal{P}} \times M_{\mathcal{P}} .
$$

In addition to the requirement that the partition be conforming, it is customary to impose a shape regularity condition on the elements $K \in \mathcal{P}$ whereby the ratio of the diameter of the element to the diameter of the largest ball that may be inscribed in $K$ remains uniformly bounded over all elements in all partitions. However, it is desirable to allow partitions $\mathcal{P}$ in which the elements are highly stretched, or anisotropic, such as shown in Figure 1. With this in mind, we suppose that an initial shape regular conforming partition $\mathcal{P}_{0}$ is given and let $\boldsymbol{c} \in \mathcal{C}$ be a collection of vertices in $\mathcal{P}_{0}$. An anisotropically refined partition $\mathcal{P}$ is permitted whereby the elements $K$ in the initial partition $\mathcal{P}_{0}$ containing a vertex $c \in \mathcal{C}$ are replaced by corner patches as shown in Figure 1 in which the conformity of the refined partition $\mathcal{P}$ has been maintained by inserting edge patches as appropriate. For $c \in \mathcal{C}$ let $\Omega_{\boldsymbol{c}}$ denote the subdomain defined by $\Omega_{c}=\cup\left\{K \in \mathcal{P}_{0}: c \in K\right\}$ and let $\omega_{c}=\cup\{K \in$ $\mathcal{P}: c \in K\}$. 

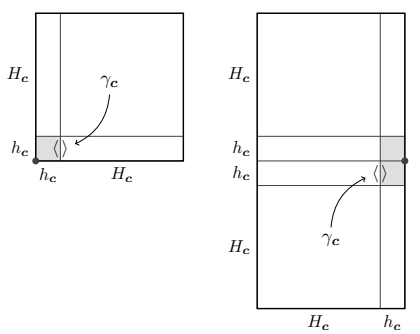
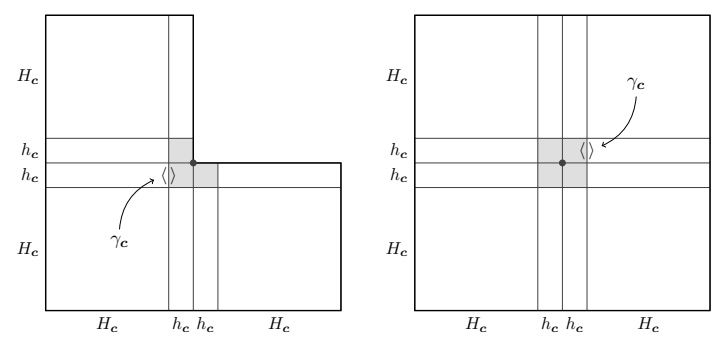

FiguRE 2. Typical anisotropically refined corner patches $\Omega_{c}$ with the corresponding subsets $\omega_{c}$ shown shaded. On the left: a single corner patch.

We may (by performing one or more uniform subdivisions of the initial partitioning $\mathcal{P}_{0}$ prior to inserting corner patches) assume that the regions $\Omega_{c}$ are disjoint, simply connected subregions of the type indicated in Figure 1. The set of elements $K \in \Omega_{c} \cap \mathcal{P}_{0}$ is shape regular and quasi-uniform of size $\mathcal{O}\left(H_{c}\right)$. Furthermore, the set of elements $K \in \omega_{\boldsymbol{c}} \cap \mathcal{P}$ is also shape regular and quasi-uniform of size $h_{\boldsymbol{c}}$. However, the refined mesh contains anisotropic elements of aspect ratio $\varrho_{c}=h_{\boldsymbol{c}} / H_{\boldsymbol{c}} \ll 1$ in each patch $\Omega_{c}$. With each node $\boldsymbol{c} \in \mathcal{C}$, we arbitrarily select a single inter-element edge $\gamma_{\boldsymbol{c}} \in \partial\left(\Omega_{\boldsymbol{c}} \backslash \omega_{\boldsymbol{c}}\right) \cap \partial \omega_{\boldsymbol{c}}$. The foregoing assumptions mean that the edge $\gamma_{\boldsymbol{c}}$ is shared by a shape regular element $\kappa_{c} \in \mathcal{P}$ of size $h_{c}$ and an anisotropic element $K_{c} \in \mathcal{P}$ of aspect ratio $\varrho_{c}$ as illustrated in Figure 2 .

The inclusion of stretched elements is beneficial for the resolution of local features such as boundary layers. Unfortunately, as numerical observations show, the discrete inf-sup constant is sensitive to the aspect ratio of the elements in the mesh, and the order $k$ of the underlying finite elements. A detailed analysis [3] reveals that

$$
\inf _{q \in M_{\mathcal{P}}} \sup _{\boldsymbol{v} \in \boldsymbol{V}_{\mathcal{P}}} \frac{(\operatorname{div} \boldsymbol{v}, q)_{\Omega}}{|\boldsymbol{v}|_{H^{1}(\Omega)}\|q\|_{\Omega}}=\beta_{\mathcal{P}} \geq C k^{-1 / 2} \min \{1, k \sqrt{\varrho}\}
$$

where $\varrho=\min \left\{\varrho_{c}: c \in \mathcal{C}\right\}$. Here, and throughout the remainder of this article, the letter $C$ will be used to represent a generic constant that is independent of any mesh size, aspect ratio or polynomial degree, and whose value need not be the same in any two differing instances.

The stability constant in the estimate (8) exhibits a relatively mild degeneration in the inf-sup constant as the order $k$ is increased which, however, can be alleviated by augmenting the space $\boldsymbol{V}_{\mathcal{P}}$ as described in [3]. Altogether more insidious is the dependence on the aspect ratio $\varrho$ which, for fixed order $k$, degenerates as $\sqrt{\varrho}$. Figure 3 shows the variation of the actual value of the inf-sup constant as the aspect ratio of the elements is varied for fixed polynomial order $k$, and confirms the behaviour with respect to $\varrho$ in the estimate (8).

The analysis [3] leading to (8) revealed that the degeneration of the inf-sup constant at high aspect ratio results from a relatively small number of spurious (piecewise constant) pressure modes identified with the sets $\omega_{\boldsymbol{c}}$ associated with corner patch refinements. This observation suggests that the degeneration might be alleviated through suppressing the spurious modes present in the discrete pressure space. Naturally, removing more pressure modes than is absolutely necessary to 


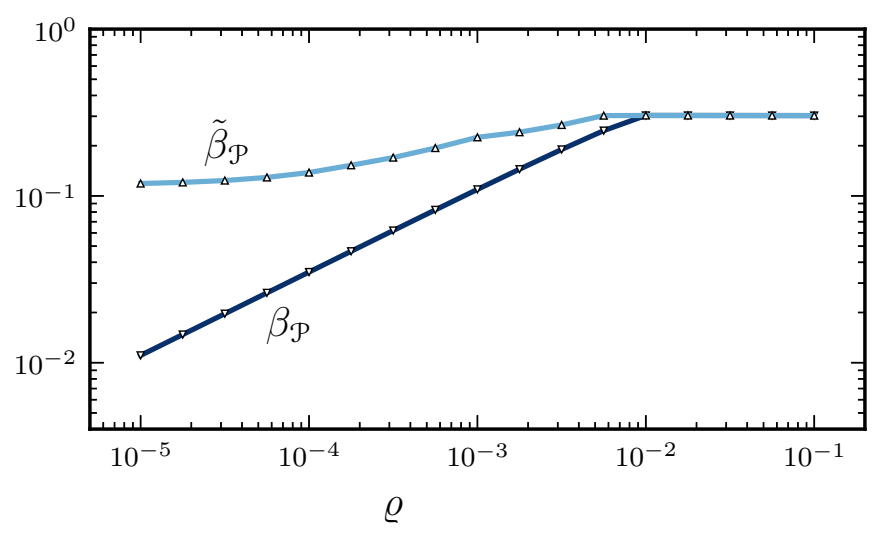

FiguRE 3. Behaviour of the inf-sup constants $\beta_{\mathcal{P}}$ and $\tilde{\beta}_{\mathcal{P}}$ with respect to the aspect ratio and polynomial degree $k=4$ on the T-mesh shown in Figure 1.

restore stability risks compromising the approximation properties of the pressure space.

The present article is concerned with resolving the problem of how to suppress the spurious pressure modes in order to restore stability yet at the same time not incurring any deterioration in the approximation properties of the reduced pressure space. Our first result gives the minimal reduction of the pressure space needed to restore stability with respect to aspect ratio:

Theorem 1. Let $\widetilde{M}_{\mathcal{P}} \subset M_{\mathcal{P}}$ denote the subspace defined by

$$
\widetilde{M}_{\mathcal{P}}=\left\{q \in M_{\mathcal{P}}: \int_{\gamma_{c}} \llbracket q \rrbracket \mathrm{d} s=0 \text { for } \boldsymbol{c} \in \mathcal{C}\right\}
$$

where $\llbracket q \rrbracket$ is the jump in the pressure $q \in M_{\mathcal{P}}$ across the edge $\gamma_{\boldsymbol{c}}$. Then, there exists a positive constant $C$, independent of the mesh size, aspect ratio and polynomial degree, such that

$$
\inf _{q \in \widetilde{M}_{\mathcal{P}}} \sup _{\boldsymbol{v} \in \boldsymbol{V}_{\mathcal{P}}} \frac{(\operatorname{div} \boldsymbol{v}, q)_{\Omega}}{|\boldsymbol{v}|_{H^{1}(\Omega)}\|q\|_{\Omega}} \geq C \tilde{\beta}_{\mathcal{P}}>0
$$

where

$$
\tilde{\beta}_{\mathcal{P}}= \begin{cases}k^{-1 / 2}, & \text { if } \mathcal{O}\left(k^{-1}\right) \leq \sqrt{\varrho} \\ k^{1 / 2} \sqrt{\varrho}, & \text { if } \mathcal{O}\left(k^{-2}\right) \leq \sqrt{\varrho} \leq \mathcal{O}\left(k^{-1}\right) \\ k^{-3 / 2}, & \text { if } \sqrt{\varrho} \leq \mathcal{O}\left(k^{-2}\right)\end{cases}
$$

and

$$
\left\|\boldsymbol{u}-\boldsymbol{u}_{\mathcal{P}}\right\|_{H^{1}(\Omega)}+\left\|p-p_{\mathcal{P}}\right\|_{\Omega} \leq C \tilde{\beta}_{\mathcal{P}}^{-2}\left(\inf _{\boldsymbol{v}_{\mathcal{P}} \in \boldsymbol{V}_{\mathcal{P}}}\left\|\boldsymbol{u}-\boldsymbol{v}_{\mathcal{P}}\right\|_{H^{1}(\Omega)}+\inf _{\tilde{q}_{\mathcal{P}} \in \tilde{M}_{\mathcal{P}}}\left\|p-\tilde{q}_{\mathcal{P}}\right\|_{\Omega}\right) .
$$

Moreover, if $p \in H^{1}(\Omega)$, then there exists a positive constant $C$ such that

$$
\inf _{\widetilde{q}_{\mathcal{P}} \in \widetilde{M}_{\mathcal{P}}}\left\|p-\widetilde{q}_{\mathcal{P}}\right\|_{\Omega}^{2} \leq C \inf _{q_{\mathcal{P}} \in M_{\mathcal{P}}}\left(\left\|p-q_{\mathcal{P}}\right\|_{\Omega}^{2}+k^{-2} \sum_{\boldsymbol{c} \in \mathcal{C}}\left|\gamma_{\boldsymbol{c}}\right|^{2}\left\|\partial\left(p-q_{\mathcal{P}}\right) / \partial n_{\boldsymbol{c}}\right\|_{\kappa_{\boldsymbol{c}} \cup K_{\boldsymbol{c}}}^{2}\right)
$$




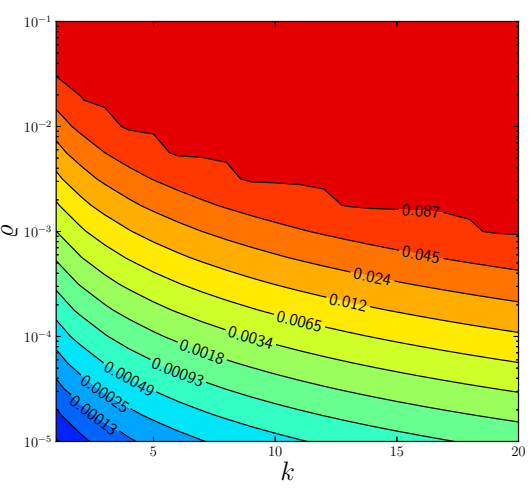

(a)
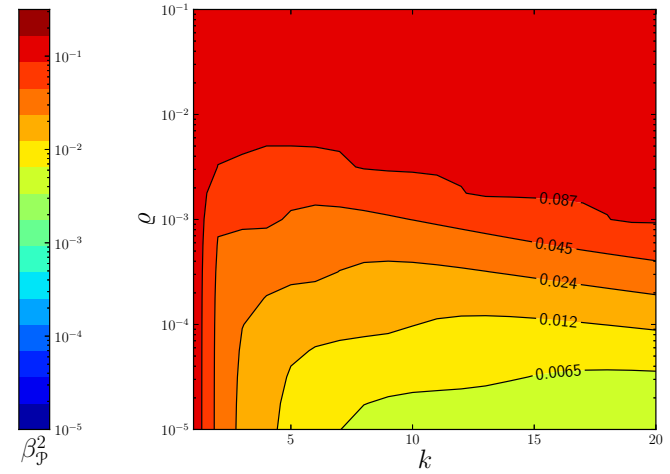

(b)

FiguRE 4. Variation of the square of the inf-sup constant with the aspect ratio and polynomial degree $k$ on the T-mesh shown in Figure 1 for (a) the mixed finite element scheme $\boldsymbol{V}_{\mathcal{P}} \times M_{\mathcal{P}}$ and (b) the new mixed finite element scheme $\boldsymbol{V}_{\mathcal{P}} \times \widetilde{M}_{\mathcal{P}}$.

where $\|\cdot\|_{\kappa_{c} \cup K_{c}}$ indicates the norm evaluated elementwise over $\kappa_{\boldsymbol{c}}$ and $K_{c}$.

Clearly $\tilde{\beta}_{\mathcal{P}} \geq \beta_{\mathcal{P}}$ since $\widetilde{M}_{\mathcal{P}} \subset M_{\mathcal{P}}$. However, more interestingly, Theorem 1 shows that the mixed finite element pair $\boldsymbol{V}_{\mathcal{P}} \times \widetilde{M}_{\mathcal{P}}$ is relatively insensitive to the aspect ratio of the elements in the sense that if $\varrho \rightarrow 0$, for fixed polynomial order $k$, then $\tilde{\beta}_{\mathcal{P}}$ remains uniformly bounded away from zero. An alternative interpretation of the result would be that if a fixed mesh is employed, containing anisotropic elements for which $\varrho \ll 1$, and convergence is sought by raising the order $k$, then the stability of the resulting method will degenerate as $\tilde{\beta}_{\mathcal{P}}=\mathcal{O}\left(k^{-3 / 2}\right)$. This compares favourably with the behaviour $\beta_{\mathcal{P}}=C k^{-1 / 2} \min \{1, k \sqrt{\varrho}\}$ for the standard scheme given by (8).

The approximation properties of the constrained pressure space $\widetilde{M}_{\mathcal{P}}$ compared with those of the unconstrained space $M_{\mathcal{P}}$ are presented in estimate (13). The only difference arises from a term involving the derivative of the pressure which generally gives rise to a lower rate of convergence but in this case is outweighed by the presence of the multiplicative factor $\left|\gamma_{c}\right| / k$. This means that the approximation properties of $\widetilde{M}_{\mathcal{P}}$ and $M_{\mathcal{P}}$ are essentially identical.

Figure 3 also shows the value of the discrete inf-sup constant for the constrained pressure space as the aspect ratio is varied for a fixed polynomial degree $k=4$. Remarkably, the simple expedient of adding a single constraint in each corner patch completely removes the degeneration of the inf-sup constant with the aspect ratio. The behaviour of the $\beta_{\mathcal{P}}^{2}$ and $\tilde{\beta}_{\mathcal{P}}^{2}$ as both the aspect ratio and the polynomial degree are varied on the mesh shown in Figure 1 is given in Figure 4. In particular, the case results shown in Figure 3 correspond to the case $k=4$ in Figure 4 . It is observed that for fixed polynomial degree $k$, the value of $\beta_{\mathcal{P}}^{2}$ degrades as $\varrho \rightarrow 0$ whilst the value of $\tilde{\beta}_{\mathcal{P}}^{2}$ remains insensitive to the value of $\varrho$. It is also seen that for $\varrho \ll 1, \beta_{\mathcal{P}}$ increases with $k$ as predicted by estimate (8) whilst $\tilde{\beta}_{\mathcal{P}}$ remains relatively insensitive. 
Theorem 1 shows that one can seek a mixed finite element approximation from the space $\boldsymbol{V}_{\mathcal{P}} \times \widetilde{M}_{\mathcal{P}}$ instead of $\boldsymbol{V}_{\mathcal{P}} \times M_{\mathcal{P}}$. Alternatively, one can impose the constraint weakly through the use of a stabilised finite element scheme as follows. Let

$$
B_{s}(\boldsymbol{u}, p ; \boldsymbol{v}, q)=B(\boldsymbol{u}, p ; \boldsymbol{v}, q)-S(p ; q)
$$

where the stabilisation term is given by

$$
S(p ; q)=\frac{1}{k^{2}} \sum_{c \in \mathcal{C}} \int_{\gamma_{c}} \llbracket p \rrbracket \mathrm{d} s \cdot \int_{\gamma_{c}} \llbracket q \rrbracket \mathrm{d} s .
$$

The stabilised finite element approximation consists of seeking $\left(\boldsymbol{u}_{\mathcal{P}}^{s}, p_{\mathcal{P}}^{s}\right) \in \boldsymbol{V}_{\mathcal{P}} \times M_{\mathcal{P}}$ such that

$$
B_{s}\left(\boldsymbol{u}_{\mathcal{P}}^{s}, p_{\mathcal{P}}^{s} ; \boldsymbol{v}, q\right)=(\boldsymbol{f}, \boldsymbol{v})_{\Omega} \text { for all }(\boldsymbol{v}, q) \in \boldsymbol{V}_{\mathcal{P}} \times M_{\mathcal{P}} .
$$

The second main result of the present work shows that the stabilised finite element scheme is robust with respect to the aspect ratio of the elements and produces an approximation that satisfies an error bound of the same type to the mixed finite element scheme using the constrained space $\widetilde{M}_{\mathcal{P}}$ :

Theorem 2. For all $(\boldsymbol{w}, r) \in \boldsymbol{V}_{\mathcal{P}} \times M_{\mathcal{P}}$, there holds

$$
\sup _{(\boldsymbol{v}, q) \in \boldsymbol{V}_{\mathcal{P}} \times M_{\mathcal{P}}} \frac{B_{s}(\boldsymbol{w}, r ; \boldsymbol{v}, q)}{\|(\boldsymbol{v}, q)\|} \geq \mu_{\mathcal{P}}\|(\boldsymbol{w}, r)\|,
$$

where $\mu_{\mathcal{P}}=C \tilde{\beta}_{\mathcal{P}}^{2}$ and $\|(\boldsymbol{v}, q)\|^{2}=|\boldsymbol{v}|_{H^{1}(\Omega)}^{2}+\|q\|_{\Omega}^{2}$. Moreover, if $p \in H^{1}(\Omega)$, then there exists a positive constant $C$, independent of the mesh size, aspect ratio and polynomial degree, such that

$$
\begin{aligned}
& \left\|\left(\boldsymbol{u}-\boldsymbol{u}_{\mathcal{P}}^{s}, p-p_{\mathcal{P}}^{s}\right)\right\| \leq\left(1+C \tilde{\beta}_{\mathcal{P}}^{-2}\right) \\
& \quad \inf _{\left(\boldsymbol{v}_{\mathcal{P}}, p_{\mathcal{P}}\right) \in \boldsymbol{V}_{\mathcal{P}} \times M_{\mathcal{P}}}\left\{\left\|\left(\boldsymbol{u}-\boldsymbol{v}_{\mathcal{P}}, p-q_{\mathcal{P}}\right)\right\|+k^{-1}\left(\sum_{\boldsymbol{c} \in \mathcal{C}}\left|\gamma_{\boldsymbol{c}}\right|^{2}\left\|\partial\left(p-q_{\mathcal{P}}\right) / \partial n_{\boldsymbol{c}}\right\|_{\kappa_{\boldsymbol{c}} \cup K_{\boldsymbol{c}}}^{2}\right)^{1 / 2}\right\} .
\end{aligned}
$$

The a priori error bound (18) for the stabilised scheme is consistent with the corresponding bound for the mixed finite element scheme using the pair $\boldsymbol{V}_{\mathcal{P}} \times \widetilde{M}_{\mathcal{P}}$ resulting from combining estimates (12) and (13).

Figure 5 shows the inf-sup constant $\mu_{\mathcal{P}}$ of estimate (17) in Theorem 2. Comparison with the results shown in Figure 4(b) confirms that the constant in (17) indeed varies as $\tilde{\beta}_{\mathcal{P}}^{2}$.

The practical implementation of the constraint in (9) is readily accomplished by using a local basis for the pressure in which the average value of the pressure on the edge $\gamma_{\boldsymbol{c}}$ is a degree of freedom. One would then simply assign the same global degree of freedom number to the corresponding local degrees of freedom in adjacent elements.

\section{Auxiliary Lemmas}

In this section, we collect various technical results used in the proofs of Theorems 1 and 2: 


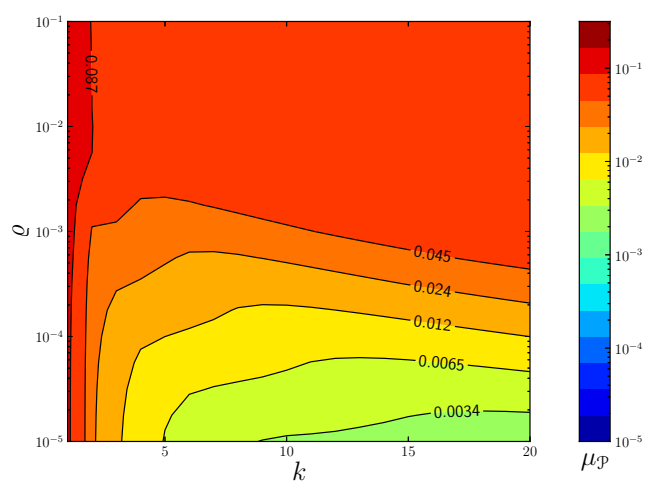

FiguRE 5. Behaviour of the inf-sup constant $\mu_{\mathcal{P}}$ appearing in (17) of the stabilised mixed finite element scheme $\boldsymbol{V}_{\mathcal{P}} \times M_{\mathcal{P}}$ on the T-mesh shown in Figure 1.

Lemma 1. Let $H, h>0$ and $k \in \mathbb{N}$. Then, there exists a univariate polynomial $\ell_{k-1}$ of degree $k-1$ such that $\ell_{k-1}(1)=1$ and

$$
\int_{-1}^{1} \ell_{k-1}(s)^{2} \mathrm{~d} s=\frac{2}{k^{2}}=\int_{-1}^{1} \ell_{k-1}(s) \mathrm{d} s .
$$

Moreover, for all $q \in \mathbb{P}_{k-1}((0, H) \times(0, h))$,

$$
\frac{1}{h} \int_{0}^{h} q(0, y)^{2} \mathrm{~d} y \leq \frac{k^{2}}{H h} \int_{0}^{h} \int_{0}^{H} q(x, y)^{2} \mathrm{~d} x \mathrm{~d} y .
$$

Proof. Let $\ell_{k-1}=2 k^{-2} \sum_{j=0}^{k-1}\left(j+\frac{1}{2}\right) P_{j}$, where $P_{j}$ is the Legendre polynomial of degree $j$. Standard properties of Legendre polynomials readily show that $\ell_{k-1}$ satisfies the conditions claimed.

Moreover, let $f$ be a univariate polynomial of degree $k-1$, and write $f=$ $\sum_{j=0}^{k-1} f_{j} P_{j}$. Again using standard properties of Legendre polynomials, we obtain

$$
f(1)^{2}=\left(\sum_{j=0}^{k-1} f_{j}\right)^{2} \leq \sum_{j=0}^{k-1}\left(j+\frac{1}{2}\right) \cdot \sum_{j=0}^{k-1}\left(j+\frac{1}{2}\right)^{-1} f_{j}^{2}=\frac{k^{2}}{2} \int_{-1}^{1} f(s)^{2} \mathrm{~d} s .
$$

Let $q$ be as in the statement of the lemma and, for fixed $y \in[0, h]$, insert $f(s)=$ $q((1+s) H / 2, y), s \in(-1,1)$ into the above inequality. Integrating the resulting inequality over $y \in[0, h]$ gives the second result.

For the next result, we recall that $\langle q\rangle_{\omega}$ denotes the mean value of $q$ over $\omega \subset \Omega$ :

Lemma 2. Let $\boldsymbol{c} \in \mathcal{C}$ and suppose edge $\gamma_{\boldsymbol{c}}$ separates elements $\kappa_{\boldsymbol{c}}$ and $K_{\boldsymbol{c}}$. Then, there exists a constant $C$ such that for both choices $K=\kappa_{\boldsymbol{c}}$ and $K=K_{\boldsymbol{c}}$ there hold:

$$
\left|\langle q\rangle_{\gamma_{c}}\right| \leq C\left|\gamma_{\boldsymbol{c}}\right|^{-1}\|q\|_{K}+\left\|\partial q / \partial n_{\boldsymbol{c}}\right\|_{K} \text { for all } q \in H^{1}(K)
$$

where $\boldsymbol{n}_{\boldsymbol{c}}$ is the unit normal on $\gamma_{\boldsymbol{c}}$; and

$$
\left|\left\langle q_{\mathcal{P}}\right\rangle_{\gamma_{c}}\right| \leq C k\left|\gamma_{\boldsymbol{c}}\right|^{-1}\left\|q_{\mathcal{P}}\right\|_{K} \text { for all } q_{\mathcal{P}} \in M_{\mathcal{P}} .
$$


Proof. Let $K$ be as in the statement. First suppose $q \in H^{1}(K)$. A scaled trace inequality $[2]$ gives

$$
\left|\gamma_{\boldsymbol{c}}\right|^{-1}\|q\|_{\gamma_{\boldsymbol{c}}}^{2} \leq C\|q\|_{K}\left\{\frac{1}{|K|}\|q\|_{K}+\frac{d_{K}}{|K|}\left\|\partial q / \partial n_{\boldsymbol{c}}\right\|_{K}\right\}
$$

where $d_{K}=\operatorname{diam}(K) \leq C\left|\gamma_{\boldsymbol{c}}\right|^{-1}|K|$ and $1 /|K| \leq C\left|\gamma_{\boldsymbol{c}}\right|^{-2}$. Hence,

$$
\langle q\rangle_{\gamma_{c}}^{2} \leq\left|\gamma_{\boldsymbol{c}}\right|^{-1}\|q\|_{\gamma_{c}}^{2} \leq C\left|\gamma_{\boldsymbol{c}}\right|^{-1}\|q\|_{K}\left\{\left|\gamma_{\boldsymbol{c}}\right|^{-1}\|q\|_{K}+\left\|\partial q / \partial n_{\boldsymbol{c}}\right\|_{K}\right\}
$$

which implies the first assertion.

Turning to the second assertion, suppose $q_{\mathcal{P}} \in M_{\mathcal{P}}$. Without loss of generality, we suppose that the edge $\gamma_{\boldsymbol{c}}$ separates an element $\kappa_{\boldsymbol{c}}$ of size $h_{\boldsymbol{c}} \times h_{\boldsymbol{c}}$ from an anisotropic element $K_{\boldsymbol{c}}$ of size $H_{\boldsymbol{c}} \times h_{\boldsymbol{c}}$, with $h_{\boldsymbol{c}}<H_{\boldsymbol{c}}$. Applying Lemma 1 , we conclude that

$$
\left\langle q_{\mathcal{P}}\right\rangle_{\gamma_{\boldsymbol{c}}}^{2} \leq\left|\gamma_{\boldsymbol{c}}\right|^{-1}\|q\|_{\gamma_{c}}^{2} \leq \frac{k^{2}}{|K|}\left\|q_{\mathcal{P}}\right\|_{K}^{2}
$$

and the estimate now follows using $1 /|K| \leq C\left|\gamma_{c}\right|^{-2}$.

Let $c \in \mathcal{C}$ and, without loss of generality, suppose that a local coordinate system is chosen in which the $\gamma_{\boldsymbol{c}}$ separates elements $\kappa_{\boldsymbol{c}}=\left(0, h_{\boldsymbol{c}}\right) \times\left(0, h_{\boldsymbol{c}}\right)$ and $K_{\boldsymbol{c}}=$ $\left(-H_{\boldsymbol{c}}, 0\right) \times\left(0, h_{\boldsymbol{c}}\right)$. Define $\chi_{\boldsymbol{c}} \in M_{\mathcal{P}}$ by the rule

$$
\chi_{\boldsymbol{c}}(x, y)= \begin{cases}\frac{1}{\left|\kappa_{\boldsymbol{c}}\right|} \ell_{k-1}\left(1-2 x / h_{\boldsymbol{c}}\right) & \text { in } \kappa_{\boldsymbol{c}} \\ -\frac{1}{\left|K_{\boldsymbol{c}}\right|} \ell_{k-1}\left(2 x / H_{\boldsymbol{c}}+1\right) & \text { in } K_{\boldsymbol{c}} \\ 0 & \text { otherwise }\end{cases}
$$

where $\ell_{k-1}$ is the polynomial defined in Lemma 1 . Simple computation and Lemma 1 reveals $\left\langle\chi_{\boldsymbol{c}}\right\rangle_{\Omega}=0$,

$$
\left\langle\llbracket \chi_{c} \rrbracket\right\rangle_{\gamma_{c}}=\frac{1}{\left|\kappa_{c}\right|}+\frac{1}{\left|K_{c}\right|}
$$

and

$$
\left\|\chi_{\boldsymbol{c}}\right\|_{\Omega}^{2}=\frac{1}{k^{2}}\left\langle\llbracket \chi_{\boldsymbol{c}} \rrbracket\right\rangle_{\gamma_{\mathbf{c}}}
$$

Consequently, we may define a mapping $\widetilde{\Pi}_{\mathcal{P}}: M_{\mathcal{P}} \rightarrow \widetilde{M}_{\mathcal{P}}$ by the rule

$$
\widetilde{\Pi}_{\mathcal{P}} q=q-\sum_{c \in \mathcal{C}} \frac{\langle\llbracket q \rrbracket\rangle_{\gamma_{c}}}{\left\langle\llbracket \chi_{c} \rrbracket\right\rangle_{\gamma_{c}}} \chi_{c} .
$$

The mapping is related to the choice of stabilisation term as follows:

Lemma 3. There exist positive constants $C_{1}, C_{2}$ such that for all $q_{\mathcal{P}} \in M_{\mathcal{P}}$,

$$
C_{1}\left\|q_{\mathcal{P}}-\widetilde{\Pi}_{\mathcal{P}} q_{\mathcal{P}}\right\|_{\Omega}^{2} \leq S\left(q_{\mathcal{P}}, q_{\mathcal{P}}\right) \leq C_{2}\left\|q_{\mathcal{P}}-\widetilde{\Pi}_{\mathcal{P}} q_{\mathcal{P}}\right\|_{\Omega}^{2} .
$$

Furthermore,

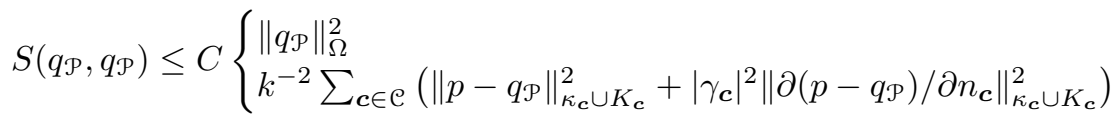

for all $p \in H^{1}(\Omega)$. 
Proof. Definition (26) gives

$$
\left\|q_{\mathcal{P}}-\widetilde{\Pi}_{\mathcal{P}} q_{\mathcal{P}}\right\|_{\Omega}^{2}=\sum_{\boldsymbol{c} \in \mathcal{C}}\left(\frac{1}{\tau_{\boldsymbol{c}}} \int_{\gamma_{\boldsymbol{c}}} \llbracket q_{\mathcal{P}} \rrbracket \mathrm{d} s\right)^{2}
$$

where $\tau_{\boldsymbol{c}}=\left|\gamma_{\boldsymbol{c}}\right|\left\langle\llbracket \chi_{\boldsymbol{c}} \rrbracket\right\rangle_{\gamma_{\boldsymbol{c}}} /\left\|\chi_{\boldsymbol{c}}\right\|_{\Omega}$. Equation (25) then implies that $\tau_{\boldsymbol{c}}^{2}=k^{2}\left|\gamma_{\boldsymbol{c}}\right|^{2}\left\langle\llbracket \chi_{\boldsymbol{c}} \rrbracket\right\rangle_{\gamma_{\boldsymbol{c}}}$. Equation (24) and the assumptions on the construction of the partition mean that there exists positive constants $c_{1}$ and $c_{2}$ for which

$$
c_{1} \leq \frac{\left|\gamma_{\boldsymbol{c}}\right|^{2}}{\left|\kappa_{\boldsymbol{c}}\right|} \leq\left|\gamma_{\boldsymbol{c}}\right|^{2}\left\langle\llbracket \chi_{\boldsymbol{c}} \rrbracket\right\rangle_{\gamma_{\boldsymbol{c}}} \leq 2 \frac{\left|\gamma_{\boldsymbol{c}}\right|^{2}}{\left|\kappa_{\boldsymbol{c}}\right|} \leq c_{2}
$$

Hence $c_{1} k^{2} \leq \tau_{\boldsymbol{c}}^{2} \leq c_{2} k^{2}$, and the first assertion follows using the definition of $S(\cdot, \cdot)$.

Using the estimate $(22)$ we obtain

$$
\left|\gamma_{\boldsymbol{c}}\right|^{2}\left\langle\llbracket q_{\mathcal{P}} \rrbracket\right\rangle_{\gamma_{c}}^{2} \leq 2\left|\gamma_{\boldsymbol{c}}\right|^{2}\left\langle\left. q_{\mathcal{P}}\right|_{\kappa_{c}}\right\rangle_{\gamma_{\boldsymbol{c}}}^{2}+2\left|\gamma_{\boldsymbol{c}}\right|^{2}\left\langle\left. q_{\mathcal{P}}\right|_{K_{\boldsymbol{c}}}\right\rangle_{\gamma_{\boldsymbol{c}}}^{2} \leq C k^{2}\left\|q_{\mathcal{P}}\right\|_{\kappa_{c} \cup K_{c}}^{2}
$$

and hence

$$
S\left(q_{\mathcal{P}}, q_{\mathcal{P}}\right)=\frac{1}{k^{2}} \sum_{c \in \mathcal{C}}\left|\gamma_{\boldsymbol{c}}\right|^{2}\left\langle\llbracket q_{\mathcal{P}} \rrbracket\right\rangle_{\gamma_{c}}^{2} \leq C\left\|q_{\mathcal{P}}\right\|_{\Omega}^{2}
$$

which proves the first part of (28). For the second part, since $p \in H^{1}(\Omega)$ we have

$$
\left\langle\llbracket q_{\mathcal{P}} \rrbracket\right\rangle_{\gamma_{c}}^{2}=\left\langle\llbracket p-q_{\mathcal{P}} \rrbracket\right\rangle_{\gamma_{c}}^{2} \leq 2\left\langle\left.\left(p-q_{\mathcal{P}}\right)\right|_{K_{c}}\right\rangle_{\gamma_{c}}^{2}+2\left\langle\left.\left(p-q_{\mathcal{P}}\right)\right|_{\kappa_{c}}\right\rangle_{\gamma_{c}}^{2}
$$

and applying estimate (21) to both of the terms on the right hand side gives

$$
\left\langle\llbracket q_{\mathcal{P}} \rrbracket\right\rangle_{\gamma_{c}}^{2} \leq C\left(\left|\gamma_{\boldsymbol{c}}\right|^{-2}\left\|p-q_{\mathcal{P}}\right\|_{\kappa_{c} \cup K_{c}}^{2}+\left\|\partial\left(p-q_{\mathcal{P}}\right) / \partial n_{\boldsymbol{c}}\right\|_{\kappa_{c} \cup K_{c}}^{2}\right) .
$$

The result now follows on combining the above estimates.

\section{Proof of Theorem 1}

We begin with a useful consequence of Theorem 4.7 in [3]:

Corollary 1. Let $M_{\mathcal{P}}^{*}=\left\{q \in M_{\mathcal{P}}:\langle q\rangle_{\omega_{c}}=0, \quad \boldsymbol{c} \in \mathcal{C}\right\}$. Then, there is a positive constant $C$, independent of any aspect ratio such that for all $q \in M_{\mathcal{P}}^{*}$, there exists $\boldsymbol{v}^{*} \in \boldsymbol{V}_{\mathcal{P}}$ satisfying:

$$
\left(\operatorname{div} \boldsymbol{v}^{*}, q\right)_{\Omega}=\|q\|_{\Omega}^{2} \text { and }\left|\boldsymbol{v}^{*}\right|_{H^{1}(\Omega)} \leq C k^{1 / 2}\|q\|_{\Omega},
$$

with $\left.\boldsymbol{v}^{*}\right|_{\omega_{c}} \in H_{0}^{1}\left(\omega_{\boldsymbol{c}}\right), \boldsymbol{c} \in \mathcal{C}$.

Proof. Let $q \in M_{\mathcal{P}}^{*}$ be given. For $c \in \mathcal{C}$, let $q_{\boldsymbol{c}}$ denote the restriction of $q$ to $\omega_{\boldsymbol{c}}$ and note that $\left\langle q_{\boldsymbol{c}}\right\rangle_{\omega_{\boldsymbol{c}}}$ vanishes. The partition $\mathcal{P}_{\boldsymbol{c}}=\left\{K \in \mathcal{P}: K \subset \omega_{\boldsymbol{c}}\right\}$ of $\omega_{\boldsymbol{c}}$ is comprised of shape regular elements, and it follows that the local mixed finite element space $\boldsymbol{V}_{\mathcal{P}_{c}} \times M_{\mathcal{P}_{c}}$ is stable independently of the local aspect ratio; i.e. there exists $\boldsymbol{v}_{\boldsymbol{c}} \in \boldsymbol{V}_{\mathcal{P}_{\boldsymbol{c}}} \cap H_{0}^{1}\left(\omega_{\boldsymbol{c}}\right)$ satisifying

$$
\left(\operatorname{div} \boldsymbol{v}_{c}, q_{c}\right)_{\omega_{c}}=\left\|q_{\boldsymbol{c}}\right\|_{\omega_{c}}^{2} \text { and }\left|\boldsymbol{v}_{\boldsymbol{c}}\right|_{H^{1}\left(\omega_{c}\right)} \leq C\left\|q_{\boldsymbol{c}}\right\|_{\omega_{c}}
$$

with $C$ independent of the aspect ratio $\varrho_{c}$. The zero extension of $\boldsymbol{v}_{\boldsymbol{c}}$ to $\Omega$ yields another function (again denoted by $\boldsymbol{v}_{\boldsymbol{c}}$ ) belonging to $\boldsymbol{V}_{\mathcal{P}}$ and satisfying the same conditions.

The function $q_{I}=q-\sum_{\boldsymbol{c} \in \mathrm{e}} q_{c}$ is supported on the subdomain $\Omega_{I}=\Omega \backslash\left(\cup_{\boldsymbol{c} \in \mathrm{e}} \omega_{\boldsymbol{c}}\right)$ and $\left\langle q_{I}\right\rangle_{\Omega_{I}}$ vanishes (since $q \in L_{0}^{2}(\Omega)$ ). Consequently, $q_{I} \in M_{\mathcal{P}_{I}}$ where $\mathcal{P}_{I}=\{K \in$ $\left.\mathcal{P}: K \in \Omega_{I}\right\}$ is a partitioning of $\Omega_{I}$. In particular, $\mathcal{P}_{I}$ is devoid of corner patches and therefore, thanks to Theorem 4.7 of [3], the mixed finite element discretisation of 
$\Omega_{I}$ is stable independently of any aspect ratio; i.e. there exists $\boldsymbol{v}_{I} \in \boldsymbol{V}_{\mathcal{P}_{I}} \cap H_{0}^{1}\left(\Omega_{I}\right)$ satisfying

$$
\left(\operatorname{div} \boldsymbol{v}_{I}, q_{I}\right)_{\Omega_{I}}=\left\|q_{I}\right\|_{\Omega_{I}}^{2} \text { and }\left|\boldsymbol{v}_{I}\right|_{H^{1}\left(\Omega_{I}\right)} \leq C k^{1 / 2}\left\|q_{I}\right\|_{\Omega_{I}}
$$

with $C$ independent of any aspect ratio. As before, the zero extension of $\boldsymbol{v}_{I}$ to $\Omega$ yields a function belonging to $\boldsymbol{V}_{\mathcal{P}}$ and satisfying the same conditions.

Let $\boldsymbol{v}^{*}=\boldsymbol{v}_{I}+\sum_{\boldsymbol{c} \in \mathcal{C}} \boldsymbol{v}_{\boldsymbol{c}} \in \boldsymbol{V}_{\mathcal{P}}$. Hence, thanks to (30)-(31) and disjoint supports, we obtain

$$
\left(\operatorname{div} \boldsymbol{v}^{*}, q\right)_{\Omega}=\left(\operatorname{div} \boldsymbol{v}_{I}, q_{I}\right)_{\Omega_{I}}+\sum_{\boldsymbol{c} \in \mathrm{C}}\left(\operatorname{div} \boldsymbol{v}_{\boldsymbol{c}}, q_{c}\right)_{\omega_{\boldsymbol{c}}}=\left\|q_{I}\right\|_{\Omega_{I}}^{2}+\sum_{\boldsymbol{c} \in \mathcal{C}}\left\|q_{\boldsymbol{c}}\right\|_{\omega_{\boldsymbol{c}}}^{2}=\|q\|_{\Omega}^{2}
$$

and

$$
\left|\boldsymbol{v}^{*}\right|_{H^{1}(\Omega)}^{2}=\left|\boldsymbol{v}_{I}\right|_{H^{1}\left(\Omega_{I}\right)}^{2}+\sum_{\boldsymbol{c} \in \mathcal{C}}\left|\boldsymbol{v}_{\boldsymbol{c}}\right|_{H^{1}\left(\omega_{c}\right)}^{2} \leq C k\left(\left\|q_{I}\right\|_{\Omega_{I}}^{2}+\sum_{\boldsymbol{c} \in \mathcal{C}}\left\|q_{\boldsymbol{c}}\right\|_{\omega_{\boldsymbol{c}}}^{2}\right) \leq C k\|q\|_{\Omega}^{2}
$$

and the result follows as claimed.

Corollary 1 shows that the instability of high aspect ratio elements originates from a single spurious pressure mode on each corner patch.

Let $q \in M_{\mathcal{P}}$ be given and let $\Pi_{\mathcal{C}} q$ be the piecewise constant function defined by the rule

$$
\Pi_{\mathcal{C}} q= \begin{cases}\langle q\rangle_{\Omega_{I}} & \text { on } \Omega_{I} \\ \langle q\rangle_{\omega_{c}} & \text { on } \omega_{\boldsymbol{c}}, \quad c \in \mathcal{C} .\end{cases}
$$

Note that $\left\langle\Pi_{\mathcal{C}} q\right\rangle_{\Omega}=\langle q\rangle_{\Omega}=0$, so that $\Pi_{\mathcal{C}} q \in M_{\mathcal{P}}$ and $q-\Pi_{\mathcal{C}} q \in M_{\mathcal{P}}^{*}$.

The next result complements Corollary 1 :

Lemma 4. There exists a positive constant $C$, independent of any aspect ratio such that

$$
\inf _{q \in \bar{M}_{\mathcal{P}}} \sup _{\boldsymbol{v} \in \boldsymbol{V}_{\mathcal{P}}} \frac{(\operatorname{div} \boldsymbol{v}, q)_{\Omega}}{|\boldsymbol{v}|_{H^{1}(\Omega)}\|q\|_{\Omega}} \geq C k^{-3 / 2}
$$

Proof. Let $q \in \widetilde{M}_{\mathcal{P}}$ be given, and decompose

$$
q=q^{*}+\Pi_{\mathcal{C}} q
$$

where $q^{*}=q-\Pi_{\mathcal{C}} q \in M_{\mathcal{P}}^{*}$. In view of Corollary 1, there exists a non-zero $\boldsymbol{v}^{*} \in \boldsymbol{V}_{\mathcal{P}}$ such that

$$
\left(\operatorname{div} \boldsymbol{v}^{*}, q^{*}\right)_{\Omega}=\left\|q^{*}\right\|_{\Omega}^{2} \text { and }\left|\boldsymbol{v}^{*}\right|_{H^{1}(\Omega)} \leq C k^{1 / 2}\left\|q^{*}\right\|_{\Omega} .
$$

Moreover, since $\left.\boldsymbol{v}^{*}\right|_{\omega_{c}} \in H_{0}^{1}\left(\omega_{\boldsymbol{c}}\right), \boldsymbol{c} \in \mathcal{C}$, we have

$$
\left(\operatorname{div} \boldsymbol{v}^{*}, \Pi_{\mathcal{C}} q\right)_{\Omega}=\langle q\rangle_{\Omega_{I}}\left(\operatorname{div} \boldsymbol{v}^{*}, 1\right)_{\Omega_{I}}+\sum_{\boldsymbol{c} \in \mathcal{C}}\langle q\rangle_{\omega_{\boldsymbol{c}}}\left(\operatorname{div} \boldsymbol{v}^{*}, 1\right)_{\omega_{\boldsymbol{c}}}=0 .
$$

Suppose for the moment that there exists a positive constant $C$ for which

$$
\|q\|_{\Omega} \leq C k\left\|q^{*}\right\|_{\Omega} .
$$

The claimed result would then follow at once since, in view of (33) and (34),

$$
\left(\operatorname{div} \boldsymbol{v}^{*}, q\right)_{\Omega}=\left(\operatorname{div} \boldsymbol{v}^{*}, q^{*}\right)_{\Omega}=\left\|q^{*}\right\|_{\Omega}^{2} \geq C k^{-1}\left\|q^{*}\right\|_{\Omega}\|q\|_{\Omega} \geq C k^{-3 / 2}\left|\boldsymbol{v}^{*}\right|_{H^{1}(\Omega)}\|q\|_{\Omega} .
$$

It therefore suffices to demonstrate (35) holds. Thanks to $q \in \widetilde{M}_{\mathcal{P}}$ and the definition of $\Pi_{\mathcal{P}} q$, we obtain

$$
\langle q\rangle_{\Omega_{I}}-\langle q\rangle_{\omega_{c}}=-\left\langle\llbracket \Pi_{\mathcal{C}} q \rrbracket\right\rangle_{\gamma_{c}}=\left\langle\llbracket q-\Pi_{\mathcal{C}} q \rrbracket\right\rangle_{\gamma_{c}}=\left\langle\llbracket q^{*} \rrbracket\right\rangle_{\gamma_{c}}, \quad \boldsymbol{c} \in \mathcal{C}
$$


and hence,

$$
\frac{1}{2} \sum_{\boldsymbol{c} \in \mathcal{C}}\left|\omega_{\boldsymbol{c}}\right|\langle q\rangle_{\omega_{\boldsymbol{c}}}^{2} \leq\langle q\rangle_{\Omega_{I}}^{2} \sum_{\boldsymbol{c} \in \mathcal{C}}\left|\omega_{\boldsymbol{c}}\right|+\sum_{\boldsymbol{c} \in \mathcal{C}}\left|\omega_{\boldsymbol{c}}\right|\left\langle\llbracket q^{*} \rrbracket\right\rangle_{\gamma_{\boldsymbol{c}}}^{2}
$$

Alternatively, equations (36) along with the relations

$$
\left|\Omega_{I}\right|\langle q\rangle_{\Omega_{I}}+\sum_{\boldsymbol{c} \in \mathcal{C}}\left|\omega_{\boldsymbol{c}}\right|\langle q\rangle_{\omega_{\boldsymbol{c}}}=|\Omega|\left\langle\Pi_{\mathcal{C}} q\right\rangle_{\Omega}=0
$$

and $\left|\Omega_{I}\right|+\sum_{\boldsymbol{c} \in \mathcal{C}}\left|\omega_{\boldsymbol{c}}\right|=|\Omega|$, give

$$
\langle q\rangle_{\Omega_{I}}=\frac{1}{|\Omega|} \sum_{c \in \mathcal{C}}\left|\omega_{c}\right|\left\langle\llbracket \llbracket q^{*} \rrbracket\right\rangle_{\gamma_{c}}
$$

Consequently,

$$
\langle q\rangle_{\Omega_{I}}^{2} \leq \frac{1}{|\Omega|}\left(\sum_{\boldsymbol{c} \in \mathcal{C}}\left|\omega_{\boldsymbol{c}}\right|\right) \frac{1}{|\Omega|} \sum_{\boldsymbol{c} \in \mathcal{C}}\left|\omega_{\boldsymbol{c}}\right|\left\langle\llbracket q^{*} \rrbracket\right\rangle_{\gamma_{\boldsymbol{c}}}^{2},
$$

and with the aid of estimate (37) and (28), we obtain

$$
\left\|\Pi_{\mathcal{C}} q\right\|_{\Omega}^{2}=\left|\Omega_{I}\right|\langle q\rangle_{\Omega_{I}}^{2}+\sum_{\boldsymbol{c} \in \mathcal{C}}\left|\omega_{\boldsymbol{c}}\right|\langle q\rangle_{\omega_{\boldsymbol{c}}}^{2} \leq C \sum_{\boldsymbol{c} \in \mathcal{C}}\left|\omega_{\boldsymbol{c}}\right|\left\langle\llbracket q^{*} \rrbracket\right\rangle_{\gamma_{\boldsymbol{c}}}^{2} \leq C k^{2} S\left(q^{*}, q^{*}\right) \leq C k^{2}\left\|q^{*}\right\|_{\Omega}^{2} .
$$

Hence,

$$
\|q\|_{\Omega}^{2}=\left\|\Pi_{\mathcal{C}} q\right\|_{\Omega}^{2}+\left\|q^{*}\right\|_{\Omega}^{2} \leq C k^{2}\left\|q^{*}\right\|_{\Omega}^{2}
$$

which implies (35) holds.

Theorem 1 is now proved as follows:

Proof. The expression for $\widetilde{\beta}_{\mathcal{P}}$ is an immediate consequence of Lemma 4 and the estimate (33) from [3]. The error bound (12) is proved in [9, Page 114].

It only remains to show the approximation theoretic properties of the space $\widetilde{M}_{\mathcal{P}}$. Let $p \in H^{1}(\Omega)$ and $q_{\mathcal{P}} \in M_{\mathcal{P}}$ be given. Define $\tilde{q}_{\mathcal{P}}=\widetilde{\Pi}_{\mathcal{P}} q_{\mathcal{P}} \in \widetilde{M}_{\mathcal{P}}$, then

$$
\frac{1}{2}\left\|p-\widetilde{q}_{\mathcal{P}}\right\|_{\Omega}^{2} \leq\left\|p-q_{\mathcal{P}}\right\|_{\Omega}^{2}+\left\|q_{\mathcal{P}}-\widetilde{\Pi}_{\mathcal{P}} q_{\mathcal{P}}\right\|_{\Omega}^{2} .
$$

By applying Lemma 3 twice, we obtain

$$
\begin{aligned}
& C_{1}\left\|q_{\mathcal{P}}-\widetilde{\Pi}_{\mathcal{P}} q_{\mathcal{P}}\right\|_{\Omega}^{2} \\
& \leq S\left(q_{\mathcal{P}}, q_{\mathcal{P}}\right) \leq C k^{-2} \sum_{\boldsymbol{c} \in \mathcal{C}}\left(\left\|p-q_{\mathcal{P}}\right\|_{\kappa_{c} \cup K_{\boldsymbol{c}}}^{2}+\left|\gamma_{\boldsymbol{c}}\right|^{2}\left\|\partial\left(p-q_{\mathcal{P}}\right) / \partial n_{\boldsymbol{c}}\right\|_{\kappa_{c} \cup K_{\boldsymbol{c}}}^{2}\right)
\end{aligned}
$$

and the result follows on collecting estimates.

\section{Proof of Theorem 2}

Lemma 5. Let $\tilde{\beta}_{\mathcal{P}}$ be as defined in (10), then

$$
\sup _{\boldsymbol{v} \in \boldsymbol{V} \mathcal{P}} \frac{(\operatorname{div} \boldsymbol{v}, q)_{\Omega}}{|\boldsymbol{v}|_{H^{1}(\Omega)}} \geq \tilde{\beta}_{\mathcal{P}}\left\|\widetilde{\Pi}_{\mathcal{P}} q\right\|_{\Omega}-\left\|q-\widetilde{\Pi}_{\mathcal{P}} q\right\|_{\Omega} .
$$

Proof. Let $q \in M_{\mathcal{P}}$, then thanks to Theorem 1 there exists a non-zero $\boldsymbol{v} \in \boldsymbol{V}_{\mathcal{P}}$ such that

$$
\begin{aligned}
\tilde{\beta}_{\mathcal{P}}|\boldsymbol{v}|_{H^{1}(\Omega)}\left\|\widetilde{\Pi}_{\mathcal{P}} q\right\|_{\Omega} \leq\left(\operatorname{div} \boldsymbol{v}, \widetilde{\Pi}_{\mathcal{P}} q\right)_{\Omega} & =\left(\operatorname{div} \boldsymbol{v}, q-\widetilde{\Pi}_{\mathcal{P}} q\right)_{\Omega}+(\operatorname{div} \boldsymbol{v}, q)_{\Omega} \\
& \leq|\boldsymbol{v}|_{H^{1}(\Omega)}\left\|q-\widetilde{\Pi}_{\mathcal{P}} q\right\|_{\Omega}+(\operatorname{div} \boldsymbol{v}, q)_{\Omega}
\end{aligned}
$$


and hence

as claimed.

$$
\sup _{\boldsymbol{v} \in \boldsymbol{V}_{\mathcal{P}}} \frac{(\operatorname{div} \boldsymbol{v}, q)_{\Omega}}{|\boldsymbol{v}|_{H^{1}(\Omega)}} \geq \tilde{\beta}_{\mathcal{P}}\left\|\widetilde{\Pi}_{\mathcal{P}} q\right\|_{\Omega}-\left\|q-\widetilde{\Pi}_{\mathcal{P}} q\right\|_{\Omega}
$$

Finally, we give the proof of Theorem 2:

Proof. Let $(\boldsymbol{w}, r) \in \boldsymbol{V}_{\mathcal{P}} \times M_{\mathcal{P}}$ be given. The proof consists of selecting a suitable pair $\boldsymbol{v} \in \boldsymbol{V}_{\mathcal{P}}$ and $q \in M_{\mathcal{P}}$ in terms of $\boldsymbol{w}$ and $r$. The equivalence (27) gives

$$
B_{s}(\boldsymbol{w}, r ; \boldsymbol{w},-r)=|\boldsymbol{w}|_{H^{1}(\Omega)}^{2}+S(r, r) \geq|\boldsymbol{w}|_{H^{1}(\Omega)}^{2}+C_{1}\left\|r-\widetilde{\Pi}_{\mathcal{P}} r\right\|_{\Omega^{2}}^{2} .
$$

By Lemma 5, there exists $\hat{\boldsymbol{w}} \in \boldsymbol{V}_{\mathcal{P}}$ such that $|\hat{\boldsymbol{w}}|_{H^{1}(\Omega)}=1$ and

$$
(\operatorname{div} \hat{\boldsymbol{w}}, r)_{\Omega} \geq \tilde{\beta}_{\mathcal{P}}\left\|\widetilde{\Pi}_{\mathcal{P}} r\right\|_{\Omega}-\left\|r-\widetilde{\Pi}_{\mathcal{P}} r\right\|_{\Omega},
$$

and hence,

$$
\begin{aligned}
B_{s}(\boldsymbol{w}, r ;-\hat{\boldsymbol{w}}, 0) & =-(\operatorname{grad} \boldsymbol{w}, \operatorname{grad} \hat{\boldsymbol{w}})_{\Omega}+(\operatorname{div} \hat{\boldsymbol{w}}, r)_{\Omega} \\
& \geq-|\boldsymbol{w}|_{H^{1}(\Omega)}+\tilde{\beta}_{\mathcal{P}}\left\|\widetilde{\Pi}_{\mathcal{P}} r\right\|_{\Omega}-\left\|r-\widetilde{\Pi}_{\mathcal{P}} r\right\|_{\Omega} .
\end{aligned}
$$

Multiplying through by $\alpha\left\|\widetilde{\Pi}_{\mathcal{P}} r\right\|_{\Omega}$, where $\alpha>0$ will be chosen later, and using the inequality $a b \leq a^{2} \tilde{\beta}_{\mathcal{P}} / 4+b^{2} / \tilde{\beta}_{\mathcal{P}}$, we obtain

$$
B_{s}\left(\boldsymbol{w}, r ;-\alpha\left\|\widetilde{\Pi}_{\mathcal{P}} r\right\|_{\Omega} \hat{\boldsymbol{w}}, 0\right) \geq \frac{\alpha}{2} \tilde{\beta}_{\mathcal{P}}\left\|\widetilde{\Pi}_{\mathcal{P}} r\right\|_{\Omega}^{2}-\frac{\alpha}{\tilde{\beta}_{\mathcal{P}}}|\boldsymbol{w}|_{H^{1}(\Omega)}^{2}-\frac{\alpha}{\tilde{\beta}_{\mathcal{P}}}\left\|r-\widetilde{\Pi}_{\mathcal{P}} r\right\|_{\Omega}^{2} .
$$

Summing (39) and (40) gives

$$
\begin{aligned}
& B_{s}\left(\boldsymbol{w}, r ; \boldsymbol{w}-\alpha\left\|\widetilde{\Pi}_{\mathcal{P}} r\right\|_{\Omega} \hat{\boldsymbol{w}},-r\right) \\
& \geq\left(1-\alpha / \tilde{\beta}_{\mathcal{P}}\right)|\boldsymbol{w}|_{H^{1}(\Omega)}^{2}+\left(C_{1}-\alpha / \tilde{\beta}_{\mathcal{P}}\right)\left\|r-\widetilde{\Pi}_{\mathcal{P}} r\right\|_{\Omega}^{2}+\frac{1}{2} \alpha \tilde{\beta}_{\mathcal{P}}\left\|\widetilde{\Pi}_{\mathcal{P}} r\right\|_{\Omega}^{2} \\
& \geq\left(\min \left(1, C_{1}\right)-\alpha / \tilde{\beta}_{\mathcal{P}}\right)\left(|\boldsymbol{w}|_{H^{1}(\Omega)}^{2}+\left\|r-\widetilde{\Pi}_{\mathcal{P}} r\right\|_{\Omega}^{2}\right)+\frac{1}{2} \alpha \tilde{\beta}_{\mathcal{P}}\left\|\widetilde{\Pi}_{\mathcal{P}} r\right\|_{\Omega}^{2} .
\end{aligned}
$$

Choosing $\alpha=\min \left(1, C_{1}\right) /\left(\tilde{\beta}_{\mathcal{P}} / 2+1 / \tilde{\beta}_{\mathcal{P}}\right)>0$, we obtain

$$
B_{s}(\boldsymbol{w}, r ; \boldsymbol{v}, q) \geq \frac{1}{2} \alpha \tilde{\beta}_{\mathcal{P}}\|(\boldsymbol{w}, r)\|\left(|\boldsymbol{w}|_{H^{1}(\Omega)}^{2}+\left\|r-\widetilde{\Pi}_{\mathcal{P}} r\right\|_{\Omega}^{2}+\left\|\widetilde{\Pi}_{\mathcal{P}} r\right\|_{\Omega}^{2}\right)^{1 / 2}
$$

where $\boldsymbol{v}=\boldsymbol{w}-\alpha\left\|\widetilde{\Pi}_{\mathcal{P}} r\right\|_{\Omega} \hat{\boldsymbol{w}}$ and $q=-r$. Moreover, since

$$
\frac{1}{2}\|q\|_{\Omega}^{2} \leq\left\|\widetilde{\Pi}_{\mathcal{P}} r\right\|_{\Omega}^{2}+\left\|r-\widetilde{\Pi}_{\mathcal{P}} r\right\|_{\Omega}^{2}
$$

and

we deduce that

$$
\frac{1}{2}|\boldsymbol{v}|_{H^{1}(\Omega)}^{2} \leq|\boldsymbol{w}|_{H^{1}(\Omega)}^{2}+\alpha^{2}\left\|\widetilde{\Pi}_{\mathcal{P}} r\right\|_{\Omega}^{2},
$$

$$
\|(\boldsymbol{v}, q)\| \leq C(1+\alpha)\|(\boldsymbol{w}, r)\| .
$$

In view of (41) we deduce that Theorem 2 holds with stability constant $\alpha \tilde{\beta}_{\mathcal{P}} / C(1+$ $\alpha) \geq C \tilde{\beta}_{\mathcal{P}}^{2}$.

The proof of the a priori error estimate is a variation of the usual argument. Let $\left(\boldsymbol{v}_{\mathcal{P}}, q_{\mathcal{P}}\right) \in \boldsymbol{V}_{\mathcal{P}} \times M_{\mathcal{P}}$ be given, then

$$
\left\|\left(\boldsymbol{u}-\boldsymbol{u}_{\mathcal{P}}^{s}, p-p_{\mathcal{P}}^{s}\right)\right\| \leq\left\|\left(\boldsymbol{u}-\boldsymbol{v}_{\mathcal{P}}, p-q_{\mathcal{P}}\right)\right\|+\left\|\left(\boldsymbol{u}_{\mathcal{P}}^{s}-\boldsymbol{v}_{\mathcal{P}}, p_{\mathcal{P}}^{s}-q_{\mathcal{P}}\right)\right\| .
$$

Let $\left(\boldsymbol{w}_{\mathcal{P}}, r_{\mathcal{P}}\right) \in \boldsymbol{V}_{\mathcal{P}} \times M_{\mathcal{P}}$ be non-zero. Direct computation using (16) gives

$$
B_{s}\left(\boldsymbol{u}_{\mathcal{P}}^{s}-\boldsymbol{v}_{\mathcal{P}}, p_{\mathcal{P}}^{s}-q_{\mathcal{P}} ; \boldsymbol{w}_{\mathcal{P}}, r_{\mathcal{P}}\right)=B\left(\boldsymbol{u}-\boldsymbol{v}_{\mathcal{P}}, p-q_{\mathcal{P}} ; \boldsymbol{w}_{\mathcal{P}}, r_{\mathcal{P}}\right)+S\left(q_{\mathcal{P}}, r_{\mathcal{P}}\right) .
$$


The first term is bounded by

$$
B\left(\boldsymbol{u}-\boldsymbol{v}_{\mathcal{P}}, p-q_{\mathcal{P}} ; \boldsymbol{w}_{\mathcal{P}}, r_{\mathcal{P}}\right) \leq C\left\|\left(\boldsymbol{u}-\boldsymbol{v}_{\mathcal{P}}, p-q_{\mathcal{P}}\right)\right\|\left\|\left(\boldsymbol{w}_{\mathcal{P}}, r_{\mathcal{P}}\right)\right\|
$$

whilst the second term is bounded using Cauchy-Schwarz and $(28)_{1}$ and $(28)_{2}$ to obtain

$S\left(q_{\mathcal{P}}, r_{\mathcal{P}}\right) \leq C k^{-1}\left\|r_{\mathcal{P}}\right\|_{\Omega}\left\{\sum_{\boldsymbol{c} \in \mathcal{C}}\left(\left\|p-q_{\mathcal{P}}\right\|_{\kappa_{c} \cup K_{\boldsymbol{c}}}^{2}+\left|\gamma_{\boldsymbol{c}}\right|^{2}\left\|\partial\left(p-q_{\mathcal{P}}\right) / \partial n_{\boldsymbol{c}}\right\|_{\kappa_{c} \cup K_{c}}^{2}\right)\right\}^{1 / 2}$.

Hence, using condition (17) gives

$$
\begin{aligned}
& \tilde{\beta}_{\mathcal{P}}^{2} \|\left(\boldsymbol{u}_{\mathcal{P}}^{s}-\boldsymbol{v}_{\mathcal{P}}, p_{\mathcal{P}}^{s}-q_{\mathcal{P}}\|\leq C\|\left(\boldsymbol{u}-\boldsymbol{v}_{\mathcal{P}}, p-q_{\mathcal{P}}\right) \|\right. \\
& +C k^{-1}\left\{\sum_{\boldsymbol{c} \in \mathcal{C}}\left(\left\|p-q_{\mathcal{P}}\right\|_{\kappa_{c} \cup K_{\boldsymbol{c}}}^{2}+\left|\gamma_{\boldsymbol{c}}\right|^{2}\left\|\partial\left(p-q_{\mathcal{P}}\right) / \partial n_{\boldsymbol{c}}\right\|_{\kappa_{\boldsymbol{c}} \cup K_{\boldsymbol{c}}}^{2}\right)\right\}^{1 / 2} .
\end{aligned}
$$

The result now follows from (42).

\section{Summary and Related Work}

The current work tackles the problem of alleviating the degeneration of the stability (inf-sup) constant of mixed finite element spaces when anisotropically refined elements are used. The stability of mixed finite element methods for incompressible flow on meshes containing anisotropically refined elements has attracted considerable attention in both the mathematical and engineering literature. Early articles addressing this question in the case of triangular elements include $[6,11]$ whilst [7] considers the stabilisation of mixed finite element schemes for the Navier-Stokes equations for quadrilateral elements; related work is presented in $[4,10]$. In each of the above mentioned articles, the starting point was a mixed finite element pair that was not uniformly inf-sup stable, even for shape regular elements.

Our approach is based on the underlying mixed finite element scheme $\mathbb{Q}_{k+1} \times$ $\mathbb{P}_{k-1}$, which was analysed for the case of isotropic (shape regular) meshes in $[5,14]$ and shown to be uniformly stable in both the mesh-size and the polynomial order $k$. It was shown $[12,13]$ that the element remains uniformly stable on meshes containing anisotropically refined edge patches, but numerical evidence showed that the stability degenerated with the aspect ratio if anisotropically refined corner patches are permitted. A detailed analysis [3] showed that the degeneration of the inf-sup constant is governed by the estimate (8) and, more importantly, that the degeneration arises from the presence of a single spurious pressure mode associated with each of the corner patches. Omitting the spurious pressure mode entirely would degrade the ability of the remaining pressure modes to adequately capture singular pressures in the neighbourhood of corners. The present work solves the delicate problem of constraining the admissible pressure modes sufficiently in order that the resulting mixed pair is uniformly stable with respect to aspect ratio whilst at the same time retaining virtually all of the approximation properties of the original pressure space. Remarkably, it transpires that this is not only possible, but can be achieved by merely constraining the average value in the pressure jump across a single edge in the neighbourhood of each corner patch. Moreover, it is shown how this result can be utilised to modify the underlying variational formulation 
of the discrete problem to obtain a scheme that is uniformly stable for the original (unconstrained) mixed finite element scheme, again without compromising the approximation properties of the resulting finite element approximation.

\section{REFERENCES}

[1] R. A. Adams, Sobolev spaces, Academic Press, New York-London, 1975. Pure and Applied Mathematics, Vol. 65.

[2] S. Agmon, Lectures on elliptic boundary value problems, Prepared for publication by B. Frank Jones, Jr. with the assistance of George W. Batten, Jr. Van Nostrand Mathematical Studies, No. 2, D. Van Nostrand Co., Inc., Princeton, N.J.-Toronto-London, 1965.

[3] M. Ainsworth And P. Coggins, The stability of mixed hp-finite element methods for Stokes flow on high aspect ratio elements, SIAM J. Numer. Anal., 38 (2000), pp. 1721-1761.

[4] T. Apel, T. Knopp, AND G. Lube, Stabilized finite element methods with anisotropic mesh refinement for the Oseen problem, Appl. Numer. Math., 58 (2008), pp. 1830-1843.

[5] C. Bernardi and Y. Maday, Uniform inf-sup conditions for the spectral discretisation of the stokes problem, Math. Methods Appl. Sci., 9 (1999), pp. 395-414.

[6] J. BLASCO, An anisotropic GLS-stabilized finite element method for incompressible flow problems, Comput. Methods Appl. Mech. Engrg., 197 (2008), pp. 3712-3723.

[7] M. BRAACK, A stabilized finite element scheme for the Navier-Stokes equations on quadrilateral anisotropic meshes, M2AN Math. Model. Numer. Anal., 42 (2008), pp. 903-924.

[8] G. P. GaLdi, An Introducction to the Mathematical Theory of the Navier-Stokes Equations, vol. 1, Springer, Berlin, 1994.

[9] V. Girault and P. Raviart, Finite Element Methods for Navier Stokes Equations, vol. 5 of Springer Series in Computational Mathematics, Springer-Verlag, 1986.

[10] Q. LiaO AND D. Silvester, Robust stabilized Stokes approximation methods for highly stretched grids, IMA J. Numer. Anal., 33 (2013), pp. 413-431.

[11] S. Micheletti, S. Perotto, and M. Picasso, Stabilized finite elements on anisotropic meshes: a priori error estimates for the advection-diffusion and the Stokes problems, SIAM J. Numer. Anal., 41 (2003), pp. 1131-1162 (electronic).

[12] D. SchötZau And C. Schwab, Mixed hp-finite element methods on anisotropic meshes, Math. Methods Appl. Sci., 8 (1998), pp. 787-820.

[13] D. Schötzau, C. Schwab, And R. Stenberg, Mixed hp-finite element methods on anisotropic meshes II. Hanging nodes and tensor products of boundary layer meshes, Numer. Math., 83 (1999), pp. 667-697.

[14] R. Stenberg and M. Suri, Mixed hp finite element methods for problems in elasticity and stokes flow, Numer. Math., 72 (1996), pp. 367-389.

Division of Applied Mathematics, Brown University, 182 George St, Providence Ri 02912, USA.

E-mail address: Mark_Ainsworth@brown.edu

Department of Mathematics and Statistics, University of Strathclyde, 26 Richmond Street, Glasgow G1 1XH, Scotland

E-mail address: \{gabriel.barrenechea, andreas.wachtel\}@strath.ac.uk 